Military Technical College

Kobry Elkobbah,

Cairo, Egypt

May 25-27,2010

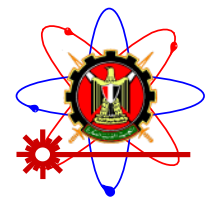

$5^{\text {th }}$ International Conference on Mathematics and Engineering Physics (ICMEP-5)

\title{
EM-21
}

\section{Application of Geometric Algebra to Generalized Hamiltonian (Birkhoffian) Mechanics}

\author{
E. T. Abou El Dahab
}

\begin{abstract}
The basic concepts of generalized Hamiltonian ( Birkhoffian ) mechanics are derived using Gemotric Algebra. Both Generalized Poisson bracket (GPB) and Generalized Lagrange bracket (GLB) are introduced. Similarly Generalized plus Poisson bracket (GPPB) and Generalized plus Lagrange bracket (GPLB) are derived which seems different from the forms by other authors. Differential constrains are derived, one of which resembles that of electromagnetic field strength.
\end{abstract}

\section{Introduction}

In this paper, the axioms of geometric Algebras as outlined in $[1,2,3,4,5,6]$ are used to formulate the generalized Hamiltonian (Birkhoffian) Mechanics. In section 2 a brief introduction to Geometric Algebra is made. In section 3 a formulation of the generalized Hamiltonian (Birkhoffian) mechanics on phase space is presented [7], and [8] and shows how to get a simple form using Geometric Algebras. The generalized Poisson bracket (BPB) is introduced in terms of geometric Algebra and in tensor forms in terms of coordinates. Jacobi' identity enables us to introduce some differential constrains on the constituents of the antisymmetric tensor defining the GPB. One of these constraints resembles the equation satisfied by the electromagnetic field strength. Similarly, the generalized Lagrange bracket (GLB) is introduced. Also, the generalized plus Poisson bracket as well as generalized plus Lagrange bracket are introduced in term of geometric Algebra and in tensor forms. In section 4 we introduce the Dirac quantization map which is a generalization of what was introduced in [11]. Dirac quantization map is used to derive the plus Poisson bracket in a form different from previous one introduced by other authors [9], [10].

\section{An introduction to Geometric Algebra}

In this study we shall introduce Geometric Algebra through the axioms outlined in $[1,2,3,4$, 5, 6]. A Geometric Algebra consists of a graded linear space whose elements are called multivectors. These are defined to have an addition, and an associative product, which is distributive.

Multivectors give geometric significance by identifying grade -1 elements as vectors. From these rules it follows that geometric product of 2 vectors $a, b$ can be defined as

$$
a b=a \cdot b+a \wedge b,
$$


where

$$
a \cdot b=1 / 2(a b+b a)=1 / 2\{a, b\},
$$

is the standard scalar, or inner, product (a real scalar), and

$$
a \wedge b=1 / 2(a b-b a)=1 / 2[a, b]^{c},
$$

is the wedge, or outer, product originally introduced by Grassmann, [1].

This decomposition extends to the geometric product of a vector with a grade $-r$ multivectors $\mathrm{A}_{\mathrm{r}}$ as,

$$
a A_{r}=a \cdot A_{r}+a \wedge A_{r},
$$

where

$$
a \cdot A_{r}=1 / 2\left(a A_{r}-(-1)^{r} A_{r} a\right),
$$

is known as the inner product, and lowers the grade of $A_{r}$ by one.

Similarly,

$$
a \wedge A_{r}=1 / 2\left(a A_{r}+(-1)^{r} A_{r} a\right),
$$

raises the grade by one.

The following identities will be used in section 3, namely,

$$
\begin{gathered}
a \cdot(b \wedge c)=(a . b) c-(a . c) b, \\
(a \wedge b) .(c \wedge d)=(b . c)(a . d)-(b . d)(a . c) .
\end{gathered}
$$

\section{The Generalized Hamiltonian (Birkhoffian) Mechanics on Phase Space}

For a mechanical system described by coordinates $\left\{\mathrm{q}_{1}, \mathrm{q}_{2}, \ldots, \mathrm{q}_{\mathrm{n}}\right\}$, we firstly define configuration space as an $\mathrm{n}$-dimensional metric space $\mathrm{R}^{\mathrm{n}}$ spanned by basic $\left\{\mathrm{e}_{\mathrm{n}}\right\}$ with

$$
e_{\mu} \cdot e_{v}=1 / 2\left\{e_{\mu}, e_{v}\right\}=1 / 2\left(e_{\mu} e_{\nu}+e_{\nu} e_{\mu}\right)=g_{\mu v},
$$

for $\mu, v=1.2, \ldots, \mathrm{n}$. This is a generalization of that given in [7] and [11]. The state of system can then be represented by vectors

$$
q=q^{\mu} e_{\mu}
$$

Vectors in configuration space generate a real Geometric Algebra $R_{n}$ with geometric product.

$$
q^{\prime} q=q^{\prime} \cdot q+q^{\prime} \wedge q
$$


Introducing the vector derivative ${ }^{\partial_{q}}$ by specifying its relation to the coordinates as follow:

$$
\partial_{q}=\partial / \partial q^{\mu} e^{\mu}
$$

where $e^{\mu}$ is the reciprocal basic for $\mathrm{R}^{\mathrm{n}}$.

Similarly, we define momentum space as n-dimensional metric space $\widetilde{R}^{n}$ spanned by basic $\left\{\tilde{e}_{\mu}\right\}_{\text {with }}$

$$
\tilde{e}_{\mu} \cdot \tilde{e}_{v}=1 / 2\left\{\tilde{e}_{\mu}, \tilde{e}_{v}\right\}=1 / 2\left(\tilde{e}_{\mu} \tilde{e}_{v}+\tilde{e}_{v} \tilde{e}_{\mu}\right)=g_{\mu v},
$$

so the momentum of our mechanical system can be expressed by vectors

$$
\widetilde{P}=P^{\mu} \tilde{e}_{\mu},
$$

and the derivative with respect to $\tilde{P}$ will be

$$
\partial \tilde{P}=\partial / \partial p^{\mu} \tilde{e}^{\mu}
$$

where $\tilde{e}^{\mu}$ is the reciprocal basic for $\tilde{R}^{n}$.

We now define the phase space $M^{2 n}$ as a direct sum

$$
M^{2 n}=R^{n} \oplus \tilde{R}^{n} .
$$

This generate the phase space (Geometric) Algebra ${ }^{M^{n}}$ which is completely defined by supplementing (3.1) and (3.5) with the relations

$$
e_{\mu} \cdot \tilde{e}_{v}=1 / 2\left\{e_{\mu}, \tilde{e}_{v}\right\}=1 / 2\left(e_{\mu} \tilde{e}_{v}+\tilde{e}_{\nu} e_{\mu}\right)=f_{\mu \nu} .
$$

Note that this is completely different from that in [7] and [11], and will give new differential constrains, namely $(3,48)$ to $(3.51)$.

Defining the symplectic bivector

$$
J=\sum_{\mu} J_{\mu},
$$

with components 2- blades

$$
J_{\mu}=1 / 2\left(e^{\mu} \wedge \tilde{e}_{\mu}+e_{\mu} \wedge \tilde{e}^{\mu}\right) . \quad \text { (no sum) }
$$

It is easy to prove that

$$
e_{\mu} . J=\tilde{e}_{\mu},
$$

and

$$
\tilde{e}_{\mu} . J=-e_{\mu},
$$

using the rules given in [1]. 
Also we can prove that

$$
J_{\mu}^{2}=-1, J^{2}=-n \text {. }
$$

using (2.7), (2.8), (3.23), and (3.24), so $J / \sqrt{n}$ represents the imaginary unit i.

Now to define the generalized Hamiltonian (Birkhoffian) mechanics on phase space, we can consider the state of our physical system by a single point $\theta$ in phase space defined by

$$
\theta=q+\tilde{p}=q^{\mu} e_{\mu}+p^{\mu} \tilde{e}_{\mu}
$$

Let

$$
\theta^{r}=\left\{\begin{array}{cc}
q^{r} & 1 \leq r \leq n \\
p^{r-n} & n<r \leq 2 n
\end{array},\right.
$$

and

$$
E_{r}=\left\{\begin{array}{cc}
e_{r} & 1 \leq r \leq n \\
\tilde{e}_{r-n} & n<r \leq 2 n
\end{array} .\right.
$$

Hence the expression (3.15) can be written as

$$
\theta=\theta^{r} E_{r}
$$

Using (3.17), (3.12), and (3.13) $\tilde{E}_{r}$ will be

$$
\tilde{E}_{r}=E_{r} . J=\left\{\begin{array}{cc}
\tilde{e}_{r} & 1 \leq r \leq n \\
-e_{r-n} & n<r \leq 2 n
\end{array} .\right.
$$

Defining the scalar product in this symplectic space by

$$
\left(E_{r}, E_{s}\right)^{s}=E_{r} . \tilde{E}_{s}=1 / 2\left\{E_{r}, \tilde{E}_{s}\right\}
$$

with matrix representation

$$
\left(E_{r}, E_{s}\right)^{s}=\Omega_{r s}=\left(\begin{array}{cc}
f_{\mu v} & -g_{\mu v} \\
g_{\mu v} & f_{\mu v}
\end{array}\right),
$$

using equations (3.1), (3. 5) (3.9), and (3. 19).

Let the inverse of $\Omega_{r s}$ be $\Omega^{r s}$ in the form

$$
\Omega^{r s}=\left(\begin{array}{cc}
f^{\mu v} & g^{\mu v} \\
-g^{\mu v} & f^{\mu v}
\end{array}\right),
$$

so we have 


$$
\begin{gathered}
g_{\mu p} \cdot g^{p v}+f_{\mu \rho} \cdot f^{\rho v}=\delta_{\mu}^{v}, \\
g_{\mu p} \cdot f^{p v}-f_{\mu \rho} \cdot g^{\rho v}=0 .
\end{gathered}
$$

The anti-symmetric matrix $\Omega_{r s}$ is the lowering matrix in phase space, so we have

$$
E_{r}=\Omega_{r s} E^{s}
$$

or

$$
\left(\begin{array}{l}
e_{\mu} \\
\tilde{e}_{\mu}
\end{array}\right)=\left(\begin{array}{cc}
f_{\mu v} & -g_{\mu v} \\
g_{\mu v} & f_{\mu v}
\end{array}\right)\left(\begin{array}{c}
\tilde{e}^{v} \\
-e^{v}
\end{array}\right),
$$

where the form of $E^{s}$ is chosen such that in case of $f_{\mu v}=0$, and $g_{\mu v}=\delta_{\mu v}$ we recover the normal forms $e_{\mu}=e^{\mu}$, and $\tilde{e}_{\mu}=\tilde{e}^{\mu}$.

From equations (3.26) we conclude that

$$
\begin{aligned}
& e_{\mu}=g_{\mu \nu} e^{v}+f_{\mu \nu} \tilde{e}^{v}, \\
& \tilde{e}_{\mu}=g_{\mu \nu} \tilde{e}^{v}-f_{\mu \nu} e^{v} .
\end{aligned}
$$

We can have similar forms of $e^{\mu}$, and $\tilde{e}^{\mu}$ as

$$
\begin{aligned}
& e^{\mu}=g^{\mu v} e_{v}-f^{\mu v} \tilde{e}_{v}, \\
& \tilde{e}^{\mu}=g^{\mu v} \tilde{e}_{v}+f^{\mu v} e_{v} .
\end{aligned}
$$

The derivative with respect to a phase space point is then given by

$$
\begin{aligned}
\partial_{\theta} & =\partial q+\partial \tilde{p} \\
& =\frac{\partial}{\partial q^{\mu}} e^{\mu}+\frac{\partial}{\partial p^{\mu}} \tilde{e}^{\mu}
\end{aligned}
$$

Also

$$
\begin{aligned}
\partial_{\tilde{\theta}} & =\partial_{\theta} . J \\
& =\frac{\partial}{\partial q^{\mu}} \tilde{e}^{\mu}-\frac{\partial}{\partial p^{\mu}} e^{\mu} .
\end{aligned}
$$

The Birkhoffian of the system is a scalar-valued function on phase space

$$
B=B(\theta, \tilde{\theta})=B(q, p)
$$

Birkhoff equation for a phase space trajectory $\theta=\theta(t)$ of the system is defined as

$$
\dot{\theta}=-\partial_{\tilde{\theta}} B
$$

which can be written in components as 


$$
\begin{aligned}
\dot{q}^{\mu} e_{\mu}+\dot{p}^{\mu} \tilde{e}_{\mu} & =-\left(\frac{\partial B}{\partial q^{\mu}} \tilde{e}^{\mu}-\frac{\partial B}{\partial p^{\mu}} e^{\mu}\right) \\
& =-\left(\frac{\partial B}{\partial q^{\mu}}\left(g^{\mu v} \tilde{e}_{v}+f^{\mu v} e_{v}\right)-\frac{\partial B}{\partial p^{\mu}}\left(g^{\mu v} e_{v}-f^{\mu v} \tilde{e}_{v}\right)\right)
\end{aligned}
$$

or

$$
\dot{q}^{\mu}=g^{\mu \nu} \frac{\partial B}{\partial p^{v}}+f^{\mu \nu} \frac{\partial B}{\partial q^{v}} \quad, \quad \dot{p}^{\mu}=-g^{\mu \nu} \frac{\partial B}{\partial q^{v}}+f^{\mu \nu} \frac{\partial B}{\partial p^{v}}
$$

Note that in the special case of Hamiltonian mechanics where $f_{\mu \nu}=0$, and $g_{\mu \nu}=\delta_{\mu \nu}$, and B replaced by $\mathrm{H}$, we recover the familiar Hamilton's equations

$$
\dot{q}^{\mu}=\delta^{\mu \nu} \frac{\partial H}{\partial p^{v}}+0 \frac{\partial H}{\partial q^{v}}=\frac{\partial H}{\partial p^{\mu}}, \quad \dot{p}^{\mu}=-\delta^{\mu \nu} \frac{\partial H}{\partial q^{v}}+0 \frac{\partial H}{\partial p^{v}}=-\frac{\partial H}{\partial q^{\mu}}
$$

The generalized Poisson bracket of two scalar-valued phase function $F(\theta), G(\theta)$ can be defined by

$$
[F, G]_{-}^{g p}=\partial_{\tilde{\theta}} F \cdot \partial_{\theta} G
$$

which reduces, using (3.1), (3.5), (3.9), (3.23), (3.24) and (3.28), to

$$
\begin{aligned}
{[F, G]_{-}^{g P} } & =f^{\mu \nu} \frac{\partial F}{\partial q^{\mu}} \frac{\partial G}{\partial q^{v}}+f^{\mu \nu} \frac{\partial F}{\partial p^{\mu}} \frac{\partial G}{\partial p^{v}}+g^{\mu \nu} \frac{\partial F}{\partial q^{\mu}} \frac{\partial G}{\partial p^{v}}-g^{\mu \nu} \frac{\partial F}{\partial p^{\mu}} \frac{\partial G}{\partial q^{v}} \\
= & \Omega^{r s} \frac{\partial F}{\partial \theta^{r}} \frac{\partial G}{\partial \theta^{s}},
\end{aligned}
$$

which is, [8], the generalized definition in terms of coordinates.

For any multi vector-valued function $M=M(\theta)$, the equation of motion is

$$
\begin{aligned}
\dot{M} & =\partial_{\theta} M \cdot \dot{\theta} \\
& =-\partial_{\theta} M \cdot \partial_{\tilde{\theta}} B \\
& =[M, B]_{-}^{g},
\end{aligned}
$$

using (3.1), (3.5), (3.9), (3.23), (3.24), (3. 28) and (3.32). Finally Birkhoff equation (3.32)can be expressed in the form

$$
\dot{\theta}=[\theta, B]_{-}^{g} .
$$

The generalized Lagrange bracket is defined as [8]

$$
\begin{aligned}
{[F, G]_{-}^{g} } & \underline{\mathrm{L}} \partial_{F} \tilde{\theta} \cdot \partial_{G} \theta \\
& =\left(\tilde{e}_{\mu} \frac{\partial q^{\mu}}{\partial F}-e_{\mu} \frac{\partial p^{\mu}}{\partial F}\right) \cdot\left(e_{v} \frac{\partial q^{v}}{\partial G}+\tilde{e}_{v} \frac{\partial p^{v}}{\partial G}\right) \\
& =-f_{\mu \nu} \frac{\partial q^{\mu}}{\partial F} \frac{\partial q^{v}}{\partial G}+g_{\mu \nu} \frac{\partial q^{\mu}}{\partial F} \frac{\partial p^{v}}{\partial G}-g_{\mu \nu} \frac{\partial p^{\mu}}{\partial F} \frac{\partial q^{v}}{\partial G}-f_{\mu \nu} \frac{\partial p^{\mu}\left(3 / p^{39}\right)}{\partial F} \frac{\partial \theta^{\prime}}{\partial G} \\
& =-\Omega_{r s} \frac{\partial \theta^{r}}{\partial F} \frac{\partial \theta^{s}}{\partial G} .
\end{aligned}
$$


The generalized plus Poisson Bracket is defined as

$$
\begin{aligned}
{[F, G]_{+}^{g P} } & =\partial_{\theta} F \cdot \partial_{\theta} G \\
& =\left(\frac{\partial F}{\partial q^{\mu}} e^{\mu}+\frac{\partial F}{\partial p^{\mu}} \tilde{e}^{\mu}\right) \cdot\left(\frac{\partial G}{\partial q^{v}} e^{v}+\frac{\partial G}{\partial p^{v}} \tilde{e}^{v}\right) \\
& =g^{\mu \nu} \frac{\partial F}{\partial q^{\mu}} \frac{\partial G}{\partial q^{v}}+g^{\mu \nu} \frac{\partial F}{\partial p^{\mu}} \frac{\partial G}{\partial p^{v}}-f^{\mu \nu} \frac{\partial F}{\partial q^{\mu}} \frac{\partial G}{\partial p^{v}}+f^{\mu \nu} \frac{\partial F}{\partial p^{\mu}} \frac{\partial G}{\partial q^{v}} \\
& =K^{r s} \frac{\partial F}{\partial \theta^{r}} \frac{\partial G}{\partial \theta^{s}},
\end{aligned}
$$

where the symmetric tensor $\mathrm{K}^{\mathrm{rs}}$ is defined as

$$
K^{r s}=\left(\begin{array}{cc}
g^{\mu v} & -f^{\mu v} \\
f^{\mu v} & g^{\mu v}
\end{array}\right) .
$$

In the special case $f_{\mu \nu}=0$, and $g_{\mu \nu}=\delta_{\mu \nu}[\mathrm{F}, \mathrm{G}]^{\mathrm{gp}}$ reduces, [11], to

$$
\frac{\partial F}{\partial q^{\mu}} \frac{\partial G}{\partial q^{\mu}}+\frac{\partial F}{\partial p^{\mu}} \frac{\partial G}{\partial p^{\mu}}
$$

obtained using the Dirac quantization map.

The special form of the generalized plus Poisson bracket (3.42) is different from the previous form by other authors $[9,10]$ which is

$$
\frac{\partial F}{\partial q^{\mu}} \frac{\partial G}{\partial p^{\mu}}+\frac{\partial F}{\partial p^{\mu}} \frac{\partial G}{\partial q^{\mu}}
$$

Similarly we can define the generalized plus Lagrange bracket as

$$
[F, G]_{+}{ }^{g L}=K_{r s} \frac{\partial \theta^{r}}{\partial F} \frac{\partial \theta^{s}}{\partial G},
$$

where the symmetric tensor $\mathrm{K}_{\mathrm{rs}}$ is the inverse of $\mathrm{K}^{\mathrm{rs}}$ defined as

$$
K_{r s}=\left(\begin{array}{cc}
g_{\mu v} & f_{\mu v} \\
-f_{\mu v} & g_{\mu v}
\end{array}\right) .
$$

The generalized Poisson bracket (3. 36) satisfies Jacobi's identity if $\Omega^{r s}(\theta)$ satisfies

$$
\Omega^{s r} \frac{\partial \Omega^{t u}}{\partial \theta^{r}}+\Omega^{t r} \frac{\partial \Omega^{u s}}{\partial \theta^{r}}+\Omega^{u r} \frac{\partial \Omega^{s t}}{\partial \theta^{r}}=0 .
$$

This identity corresponds to the following identity for the tensor $\Omega_{r s}$, namely

$$
\frac{\partial \Omega_{s t}}{\partial \theta^{r}}+\frac{\partial \Omega_{t r}}{\partial \theta^{s}}+\frac{\partial \Omega_{r s}}{\partial \theta^{t}}=0 .
$$

The last relation implies the following differential constraints on both ${ }^{g_{\mu v}}$ and $f_{\mu v}$,

$$
\begin{aligned}
& \frac{\partial f_{\mu v}}{\partial q^{\rho}}+\frac{\partial f_{v \rho}}{\partial q^{\mu}}+\frac{\partial f_{\rho \mu}}{\partial q^{v}}=0, \\
& \frac{\partial f_{\mu \nu}}{\partial p^{\rho}}+\frac{\partial f_{\nu \rho}}{\partial p^{\mu}}+\frac{\partial f_{\rho \mu}}{\partial p^{v}}=0,
\end{aligned}
$$




$$
\begin{aligned}
& \frac{\partial f_{\mu v}}{\partial q^{\rho}}+\frac{\partial g_{v \rho}}{\partial p^{\mu}}-\frac{\partial g_{\rho \mu}}{\partial p^{v}}=0 \\
& \frac{\partial f_{\mu v}}{\partial p^{\rho}}-\frac{\partial g_{v \rho}}{\partial q^{\mu}}+\frac{\partial g_{\rho \mu}}{\partial q^{v}}=0
\end{aligned}
$$

The first of these four equations is similar to that satisfied by the electromagnetic field strength.

\section{The generalized form of Dirac Quantization Map:}

As was stated before, [11], the form leading to Dirac quantization map is

$$
F \rightarrow \hat{F}=\sqrt{\frac{\hbar \sqrt{n}}{2}} \partial_{\theta} F,
$$

which represents the relation between a function $\mathrm{F}$ and its corresponding operator $\hat{F}$. This relation enables us to derive the relation between Poisson bracket of two functions and the commutator of the two corresponding operators as

$$
[\hat{F}, \hat{G}]^{c}=i \hbar[F, G]_{-}^{p} .
$$

The same procedures can be done in this case of generalized Hamiltonian (Birkhoffian) theory where the Poisson bracket is replaced by the generalized one, GPB, so (4.2) will be replaced by

$$
[\hat{F}, \hat{G}]^{c}=i \hbar[F, G]_{-}^{g p}
$$

Equation (4.3) represents the generalized Dirac quantization map.

In deriving (4.2) we have used the axioms of Geometric Algebras as well as (4.1).

In a similar manner we can deduce the form of the generalized plus Poisson bracket, (3.40), which will be a generalization of the form derived in [11], namely (3.42).

\section{5-Summaries and Conclusion:}

The usage of geometric Algebras enables us to reformulate the whole generalized Hamiltonian (Birkhoffian) mechanics in a unified and simpler algebraic form rather than the coordinates form.

The formulation of both generalized Poisson and Lagrange brackets is done in terms of Geometric Algebra. Using these Algebraic forms, we can define both the plus generalized Poisson and Lagrange brackets, and show that these forms enable us to redefine the generalized Dirac quantization map. Jacobi's identity for GPB implies that there are differential constraints on both $g_{\mu v}$ and $f_{\mu v}$ forming the anti-symmetric tensor $\Omega_{r s}$. The first of these constraints resembles the differential equation satisfied by the electromagnetic field $\operatorname{tensor}_{\mu \nu}$. The remaining three differential equations impose some constrains on both $g_{\mu v}$ and $f_{\mu v}$. These differential equations need thorough investigations for clarifying the nature of both $g_{\mu v}$ and $f_{\mu v}$. 


\section{References:}

[1] Hestenes D., "New Foundations for Classical Mechanics". D. Reidel Publishing Co., Dordrecht/ Boston, 1986.

[2] A. N. Lasenby, C.J.L. Doran, S.F. Gull, “Grassmann Calculus, Pseudo Classical Mechanics and Geometric Algebra”, Journal of mathematical Physics 34, P3683, 1993.

[3] M.Pavsic, "The Landscape of Theoretical Physics: A global View". Kluwer Academic Publishers. Dordrecht/Boston/London, 2001.

[4] P. Lounesto "Clifford Algebras and Spinors", Cambridge University Press, 2001.

[5] V. De Sabbata and B.K.Datta "Geometric Algebra and Applications to Physics", Taylor \& Francis Group, 2007.

[6] J.Vince "Geometric Algebra for Computer Graphics", Springer, 2008.

[7] Pappas R.C., "A Formulation of Hamiltonian Mechanics Using Geometrical Calculus", in Brackx F. et al. (eds.), "Clifford Algebras and Their Application in Mathematical Physics”, Kluwer Academic Publishers, 1993. 1982.

[8] R. M. Santilli, "Foundation of Theoretical Mechanics II", Springer- Verlag, Berlin,

[9] P. Droz - Vincent, Inst. Henri Poincare, sec. A, 6, 28, 1966.

[10] Wilhelm H. Franke and Andrs J. Kalany, "Symmetric Dirac Bracket in Classical Mechanics”, Journal of Mathematical Physics, Vol. II, No 5, PP 1729- 1734, 1970.

[11] E. T. Abou El Dahab, "A Formulation of Hamiltonian Mechanics Using Geometric Algebra", In Advances in Applied Clifford Algebras, Volume 10, number 2, Mexico, 2000. 